\title{
THE PALOMAR OBSERVATORY DWARF SEYFERT SURVEY
}

\author{
LUIS C. HO ${ }^{1}$, ALEXEI V. FILIPPENKO ${ }^{1}$, and WALLACE L. W. SARGENT ${ }^{2}$ \\ ${ }^{1}$ Department of Astronomy, University of California, Berkeley, CA 94720, U.S.A. \\ ${ }^{2}$ Palomar Observatory, 105-24 Caltech, Pasadena, CA 91125, U.S.A.
}

\begin{abstract}
We describe an optical, spectroscopic survey of the nuclei of the 500 brightest galaxies in the northern sky. The primary goal is to search for low-luminosity active galactic nuclei (LLAGNs) in the centers of nearby galaxies. The results of this survey will have many astrophysical applications, including quantifying the faint end of the local AGN luminosity function and estimating the contribution of LLAGNs to the X-ray background. We summarize the statistical properties of the survey, describe our methods of analysis, and present some preliminary results based on $\sim 60 \%$ of the sample.
\end{abstract}

\section{Introduction}

Although it has been recognized for some time that low-level nuclear activity is present in a substantial fraction of nearby galaxies (see, e.g., review by Keel 1985), both the quantitative assessment of the prevalence of nuclear activity and a coherent understanding of the physical nature of such activity are complicated in part by the lack of a complete set of reliable data. Most of the observational evidence for the apparent ubiquity of lowluminosity active galactic nuclei (LLAGNs) has come from optical spectroscopic surveys of nearby galaxies. (As an operational definition, we define LLAGNs as galactic nuclei having $M_{B}<-18 \mathrm{mag}$ and emission-line ratios unlike those of $\mathrm{H}$ II regions.) The accuracy of the detection technique, which involves measurement and modeling of weak emission lines, critically depends on several factors, not all of which could always be met by the surveys performed in the past. These include having data of adequate signal-to-noise ratio ( $/ \mathrm{N})$ and spectral resolution, and the proper removal of the underlying starlight which often dominates the observed spectra.

A reliable census of the space density of LLAGNs will have many important applications. For example, the local $(z \approx 0)$ luminosity function of LLAGNs is very poorly constrained at the present time (e.g., Weedman 1986). The faint end of the local AGN luminosity function is of critical importance to several astrophysical issues, including the evolution of the overall AGN luminosity function and the contribution of AGNs to the cosmic Xray background. In addition, the intrinsic low luminosity of LLAGNs, coupled with their proximity, in principle allows us to study the properties of the host galaxies in a level of detail not feasible for more luminous and/or distant AGNs. In this way, we may hope to be able to better understand the fundamental issue of what physical processes are responsible for the formation of AGNs in galaxies.

\section{The Spectroscopic Database}

In an effort to address some of the issues raised in $\S 1$, an optical spectroscopic survey of the nuclei of nearby galaxies was carried out at Palomar Observatory between 1982 and 1990 (see Filippenko and Sargent 1985, 1986 for details). Briefly, the nuclei of all galaxies with $B_{T} \leq 12.5 \mathrm{mag}$ and $\delta>0$ were observed with the Double Spectrograph (Oke and Gunn 1982) mounted on the Hale $5 \mathrm{~m}$ telescope at Palomar Observatory. This statistically complete sample consists of $\sim 500$ objects. The resulting database contains spectra of excellent quality $(\mathrm{S} / \mathrm{N} \approx 100)$ and moderate spectral resolution $(2.5-4 \AA)$ over the wavelength regions $\sim 4200-5200 \AA$ and $\sim 6200-6900 \AA$. Basic calibration steps such

T. J.-L. Courvoisier and A. Blecha: Multi-Wavelength Continuum Emission of AGN, 275-278.

(C) 1994 IAU. Printed in the Netherlands. 
as bias subtraction and flatfielding, extraction of 1-D spectra $\left(2^{\prime \prime} \times 4^{\prime \prime}\right.$ effective aperture) from 2-D images, and wavelength and flux calibration follow standard procedures. As mentioned in $\$ 1$, a crucial step in the analysis of the emission-line spectra is the removal of the starlight contamination. The method adopted closely follows that described in Ho, Filippenko, and Sargent (1993), and is illustrated in Figure 1 for NGC 4261 (also see Ford et al. in these proceedings). Note that most of the emission lines of this relatively weak-lined object can only be reliably measured after the subtraction of an appropriately scaled "template" galaxy (in this case NGC 2300) whose stellar population, stellar velocity dispersion, and metalicity closely match those of the object spectrum.

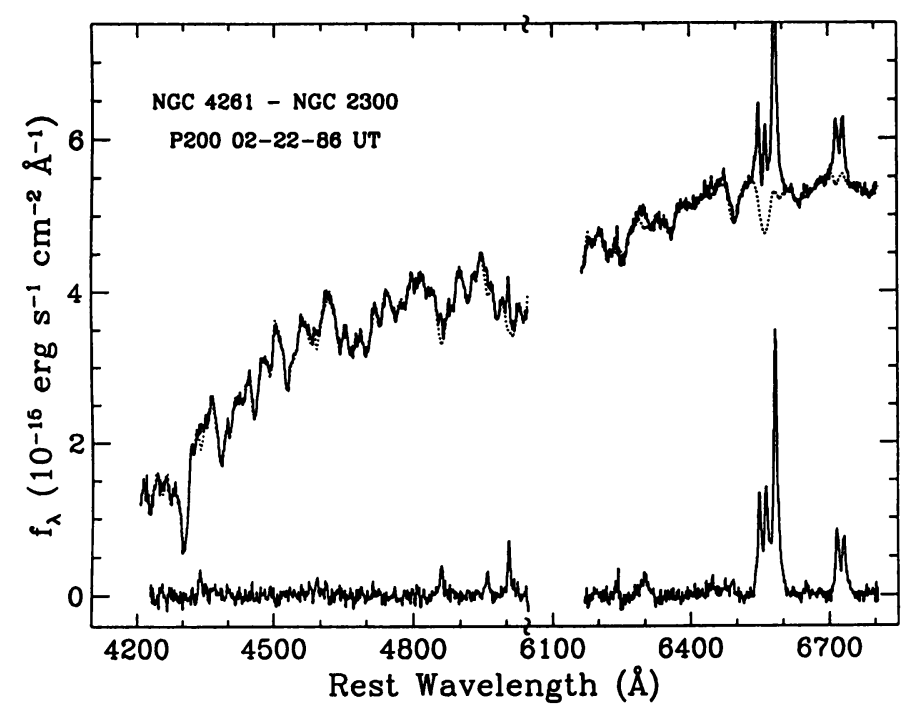

Figure 1. Illustration of starlight subtraction for NGC 4261.

\section{Preliminary Results}

From our uniformly observed, calibrated, and reduced data set, we aim to systematically quantify emission-line parameters such as fluxes and line widths, stellar velocity dispersions, and rotation curves. We will model the emission-line spectra with photoionization and shock calculations (e.g., Ho et al. 1993) in order to determine the excitation mechanism(s) operative in the nuclei, derive a luminosity function for the LiAGNs in the survey, and attempt to find correlations between the types of excitation mechanism and various global properties of the host galaxies. The database and subsequent applications thereof will be published in future papers.

In this contribution, we present a preliminary analysis of about $60 \%$ of the survey (324 galaxies), focusing on the occurrence of various classes of emission-line galaxies as a function of Hubble type. As illustrated in Figure 2, the redshift and morphological type distributions of this subsample are very similar to, and thus representative of, those of the entire survey. Conclusions we draw from the present subsample will likely be valid for 
the survey as a whole once the entire sample has been analyzed.
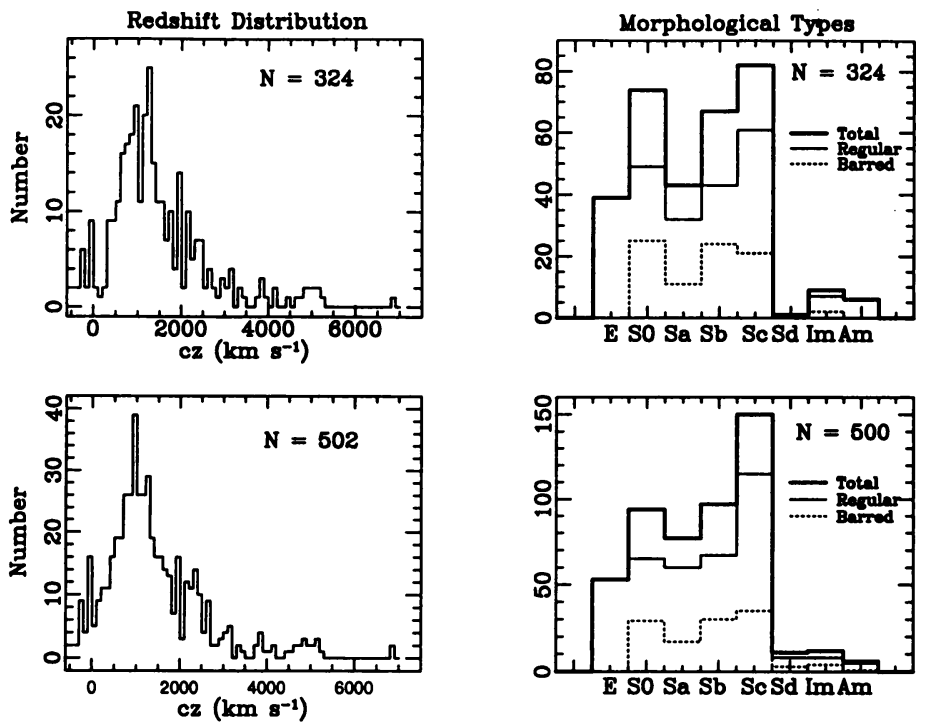

Figure 2. Redshift and morphological type distributions for objects in the subsample discussed in the text (top) and in the whole survey (bottom).

We classified the calibrated spectra containing emission lines into three "excitation classes": (1) LLAGNs, (2) H II galaxies, and (3) Seyfert 1 nuclei. The LLAGNs group includes both low-ionization nuclear emission-line regions (LINERs; Heckman 1980) and Seyfert 2 galaxies. Since we have not yet performed a careful starlight subtraction for all of the objects, we did not attempt to discriminate between the two; however, judging from the space density of Seyfert 1 galaxies and the relative space densities of Seyfert 1s and 2s (e.g., Osterbrock and Martel 1993), the vast majority of the LLAGNs should be LINERs. H II galaxies are objects whose spectra resemble those of $\mathrm{H}$ II regions and are thus galaxies with nuclei undergoing recent or current star formation. The distribution of excitation class as a function of Hubble type (Fig. 3; see also Table 1) reveals that (1) LLAGNs are nearly as numerous as $B$ II galaxies, (2) the host galaxies of LLAGNs and Seyfert 1s tend to be early-type spirals (S0-Sb), and (3) nuclear star formation (i.e., H II galaxies) occurs most frequently in later type spirals (mostly Sb-Sc).

Quantitatively, Table 1 shows that as many as $40 \%$ of all nearby galaxies may harbor an LLAGN, comparable to the fraction of galaxies whose nuclei are experiencing recent or ongoing star formation (38\%). LLAGNs make up about $50 \%$ of S0 and Sb galaxies, $~ 70 \%$ of Sa galaxies, and $\sim 60 \%$ of all spirals. Although these results are qualitatively similar to those discovered in past surveys (see Keel 1985), they are quantitatively different (and presumably more accurate). In particular, the fraction of galaxies containing mildly active nuclei appears to be even higher than previously thought. 
TABLE 1. DISTRIBUTION OF EXCITATION CLASS

\begin{tabular}{lllllllllll}
\hline \hline Class $^{1}$ & No. & $\%$ & $\mathrm{E}^{2}$ & $\mathrm{S0}$ & $\mathrm{Sa}$ & $\mathrm{Sb}$ & $\mathrm{Sc}$ & $\mathrm{Sd}$ & $\mathrm{Im}$ & $\mathrm{Am}$ \\
\hline $\mathrm{L}$ & 128 & 40 & 33 & 49 & 70 & 49 & 17 & 0 & 11 & 17 \\
$\mathrm{H}$ & 122 & 38 & 3 & 5 & 16 & 45 & 80 & 100 & 89 & 83 \\
$\mathrm{~S}$ & 8 & 3 & 0 & 4 & 5 & 4 & 0 & 0 & 0 & 0 \\
$\mathrm{~T}$ & 63 & 19 & 64 & 42 & 9 & 2 & 3 & 0 & 0 & 0
\end{tabular}

' $\mathrm{L}=$ LLAGNs, $\bar{H}=\mathrm{H}$ II galaxies, $\mathrm{S}=$ Seyfert 1 galaxies, and $\mathrm{T}$ = "template" galaxies.

${ }^{2}$ Numbers listed under each Hubble type are percentages.

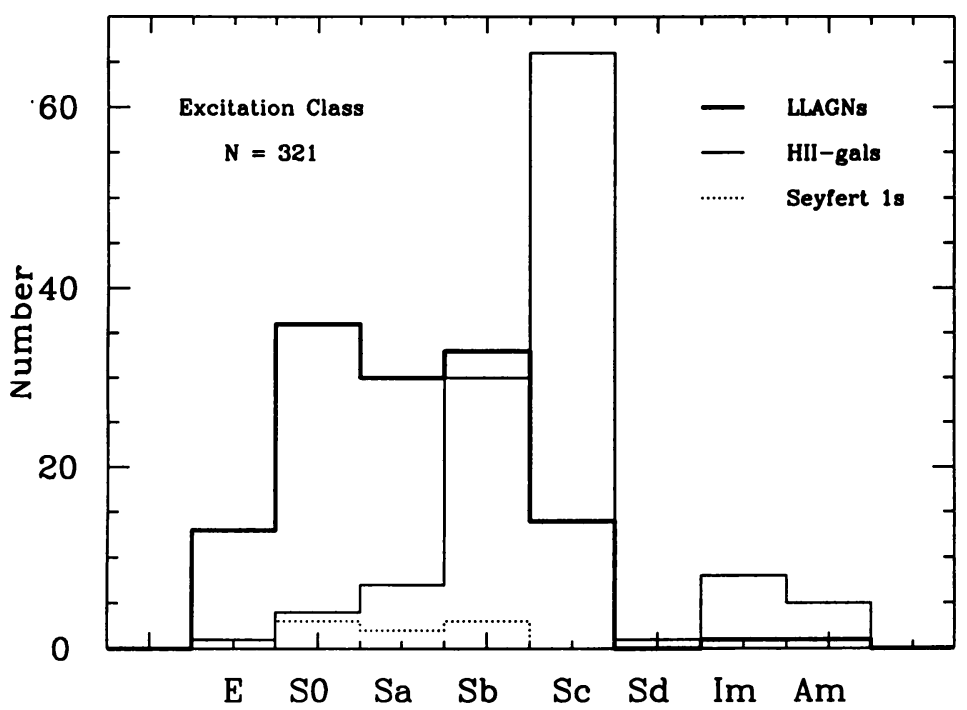

Figure 3. Distribution of excitation class as a function of Hubble type.

\section{References}

Filippenko, A. V., and Sargent, W. L. W. 1985, Ap. J. Suppl., 57, 503

Filippenko, A. V., and Sargent, W. L. W. 1986, in Structure and Evolution of Active Galactic Nuclei, ed. G. Giuricin et al. (Dordrecht: Reidel), p. 21

Heckman, T. M. 1980, Astr. Ap., 87, 152

Ho, L. C., Filippenko, A. V., and Sargent, W. L. W. 1993, Ap. J., in press

Keel, W. C. 1985, in Astrophysics of Active Galaxies and Quasi-Stellar Objects, ed. J. S.

Miller (Mill Valley, CA: Univ. Science Books), p. 1

Oke, J. B., and Gunn, J. E. 1982, Pub. A.S.P., 94, 586

Osterbrock, D. E., and Martel, A. 1993, Ap. J., 414, 552

Weedman, D. W. 1986, in Structure and Evolution of Active Galactic Nuclei, eds. G.

Giuricin et al. (Dordrecht: Reidel), p. 215 